\title{
Event-Triggered Bipartite Consensus of Single-Integrator Multi-Agent Systems with Measurement Noise
}

\author{
Cui-Qin Ma $\mathbb{D D}^{1},{ }^{1}$ Yun-Bo Zhao, ${ }^{2}$ and Wei-Guo Sun ${ }^{1}$ \\ ${ }^{1}$ School of Mathematical Sciences, Qufu Normal University, Qufu 273165, Shandong, China \\ ${ }^{2}$ College of Information Engineering, Zhejiang University of Technology, Hangzhou 310023, China \\ Correspondence should be addressed to Cui-Qin Ma; cuiqinma@amss.ac.cn
}

Received 29 May 2018; Accepted 22 July 2018; Published 6 August 2018

Academic Editor: Yong Chen

Copyright (C) 2018 Cui-Qin Ma et al. This is an open access article distributed under the Creative Commons Attribution License, which permits unrestricted use, distribution, and reproduction in any medium, provided the original work is properly cited.

\begin{abstract}
Event-triggered bipartite consensus of single-integrator multi-agent systems is investigated in the presence of measurement noise. A time-varying gain function is proposed in the event-triggered bipartite consensus protocol to reduce the negative effects of the noise corrupted information processed by the agents. Using the state transition matrix, Itô formula, and the algebraic graph theory, necessary and sufficient conditions are given for the proposed protocol to yield mean square bipartite consensus. We find that the weakest communication requirement to ensure the mean square bipartite consensus under event-triggered protocol is that the signed digraph is structurally balanced and contains a spanning tree. Numerical examples validated the theoretical findings where the system shows no Zeno behavior.
\end{abstract}

\section{Introduction}

Recent years have witnessed the great achievements in studying the consensus problem of multi-agent systems (MASs) which has broad applications in various fields [18]. We notice that in these mentioned works interactions among agents are all assumed to be cooperative to achieve consensus. However, it is very natural to see, in many real examples, that in MASs some agents cooperate while others compete, and MASs with competitive interactions can introduce more complex behaviors. To quantitatively model such a scenario, the concept of bipartite consensus, i.e., agents agree on a certain quantity with the equal modulus but different signs, has been proposed [9], and many achievements have been made [9-18]. In [9], for singleintegrator MASs, a linear feedback protocol is designed and under the assumption that the communication topology $\mathscr{G}$ is strongly connected, the MAS is proved to achieve bipartite consensus if and only if $\mathscr{G}$ is structurally balanced. Then, in [10], the communication condition in [9] is relaxed to containing a spanning tree. In [11], the communication topology in [9] is extended to the time-varying case.
It is worth noting that the above literatures mainly focus on continuous feedback protocols, where the agent state is monitored continuously and its controller is updated all the time. However, updating the controller in real-time easily increases the computational burden. Therefore, reducing the update frequency for a trade-off between the system performance and the resource usage is usually desired. This requirement then naturally brings event-triggered schemes into consideration, which updates only at some predetermined discrete time instants. Event-triggered techniques have already been widely used in traditional consensus problems of MASs [19-27]. For example, a self-triggered protocol is proposed in [19] and a decentralized eventtriggered protocol ensuring average consensus is proposed in [20] for single-integrator MASs, time-dependent triggering functions are investigated in [24] for second-order MASs, and event-triggered consensus problems are considered in [25, 26] for general linear systems, just name a few. Despite these achievements, event-triggered protocols have not been well studied for bipartite consensus [28,29], which thus motivates the present study.

In another parallel line, measurement noise is unavoidable in practice, making the investigation on 
the event-triggered bipartite consensus of MASs with noise even interesting. In fact, studies on bipartite consensus with measurement noise can be found in $[13,16-18]$, which are however all with time-triggered controllers. Event-triggered bipartite consensus for MASs with measurement noise still remains to tackle.

In this paper, we investigate event-triggered bipartite consensus for single-integrator MASs with measurement noise. A time-varying control gain is introduced into the event-triggered protocols, leading to a time-varying closedloop system. With the help of the state transition matrix and stochastic analysis theory, the closed-loop system is analyzed. Necessary and sufficient conditions for the system to achieve mean square bipartite consensus based on event-triggered protocols are given. We find that the communication topology being structurally balanced and containing a spanning tree are necessary and sufficient for ensuring a mean square bipartite consensus based on event-triggered protocols.

Organization. Section 2 gives the algebraic graph preliminaries and the problem in question. Section 3 contains the main results of the paper. Section 4 applies the results to examples of MASs with six agents. Section 5 closes this paper.

Notations. $R^{n \times m}$ represents the real matrix of $n \times m$ order. 0 denotes vector or matrix whose elements are $0 . \mathbf{1}_{n}$ represents column vector whose elements are $1 . \operatorname{sgn}(\cdot)$ represents the sign function. $\otimes$ represents Kronecker product. For a given matrix or vector $X, X^{T}$, and $\|X\|$ represent the transpose and European norm of $X$, respectively. $\|X\|_{F},\|X\|_{1}$, and $\|X\|_{\infty}$ represent the Frobenius norm, 1-norm, and $\infty$-norm, respectively. $\operatorname{Re}(\lambda)$ is the real part of $\lambda$.

\section{Problem Statement}

The communication relations among $N$ agents are described by the signed digraph $\mathscr{G}=(\mathscr{V}, \mathscr{E}, \mathscr{A})$, where $\mathscr{V}=\{1, \ldots, N\}$ and $\mathscr{E} \subseteq \mathscr{V} \times \mathscr{V}$ represent the node set and the edge set, respectively. $\mathscr{A}=\left(a_{i j}\right) \in R^{N \times N}$, where $a_{i j}>0$ and $a_{i j}<0$ represent cooperation and competition between agents $i$ and $j$, respectively. $a_{i j} \neq 0 \Longleftrightarrow(j, i) \in \mathscr{E}$. We assume that $a_{i i}=0$ and $a_{i j} a_{j i} \geq 0, \forall i, j \in \mathscr{V} . \mathscr{L}=\mathscr{C}_{r}-\mathscr{A}$ is the Laplacian matrix of $\mathscr{G}$, where $\mathscr{C}_{r}=\operatorname{diag}\left(\sum_{j=1}^{N}\left|a_{1 j}\right|, \ldots, \sum_{j=1}^{N}\left|a_{N j}\right|\right)$. A signed digraph $\mathscr{G}=(\mathscr{V}, \mathscr{E}, \mathscr{A})$ is said structurally balanced if $\mathscr{V}$ can be divided into two subsets $\mathscr{V}_{1}, \mathscr{V}_{2}, \mathscr{V}_{1} \cup \mathscr{V}_{2}=\mathscr{V}, \mathscr{V}_{1} \cap \mathscr{V}_{2}=$ $\varnothing$, such that $a_{i j} \geq 0, \forall i, j \in \mathscr{V}_{p}(p \in\{1,2\})$, and $a_{i j} \leq 0$, $\forall i \in \mathscr{V}_{p}, j \in \mathscr{V}_{q}(p \neq q, p, q \in\{1,2\})$. It is said structurally unbalanced otherwise.

Lemma 1 (see [12]). If $\mathscr{G}$ is structurally balanced, Laplacian $\mathscr{L}$ of $\mathscr{G}$ has at least one zero eigenvalue and all of the nonzero eigenvalues have positive real parts. Furthermore, $\mathscr{L}$ has only one zero eigenvalue if and only if $\mathscr{G}$ has a spanning tree.

\section{Consider a MAS described by}

$$
\dot{x}_{i}(t)=u_{i}(t), \quad i=1, \ldots, N,
$$

where $x_{i}(t) \in R^{n}$ is the state of the $i$ th agent and $u_{i}(t) \in R^{n}$ is the control input. A signed digraph $\mathscr{G}=(\mathscr{V}, \mathscr{E}, \mathscr{A})$ is used to describe interactions among the $N$ agents.
Since communication is often disturbed by measurement noise, we assume the $i$ th agent receives information from its neighbors with measurement noise $x_{j}(t)+\varphi_{j i}(t), j \in$ $\mathcal{N}_{i}, i=1, \ldots, N$. In order to reduce the frequency of controller updates, we design the following event-triggered protocol for the $i$ th agent:

$$
\begin{gathered}
u_{i}(t)=b(t)\left[\sum_{j=1}^{N}\left|a_{i j}\right|\left(\operatorname{sgn}\left(a_{i j}\right) x_{j}\left(t_{k}\right)-x_{i}\left(t_{k}\right)\right)\right. \\
\left.+\sum_{j=1}^{N} a_{i j} \varphi_{j i}(t)\right], \quad \forall t \in\left[t_{k}, t_{k+1}\right),
\end{gathered}
$$

where $i=1, \ldots, N, k=0,1, \ldots, b(t)>0$ is a piecewise continuous function. $\left\{\varphi_{j i}(t)\right\}$ is $n$ dimensional independent standard white noise.

Remark 2. As far as we know the existing results $[28,29]$ for event-triggered bipartite consensus did not consider measurement noise. Here, we take noise into consideration. If we take $b(t) \equiv 1$, then (2) is reduced to the protocols in $[28,29]$ without measurement noise.

Let $X(t)=\left(x_{1}^{T}(t), \ldots, x_{N}^{T}(t)\right)^{T}$ and $J=\operatorname{diag}\left(\varsigma_{1}^{T}(t), \ldots\right.$, $\left.\varsigma_{N}^{T}(t)\right)^{T}$ be $N \times N^{2}$ dimensional block diagonal matrix, where $\varsigma_{i}^{T}(t)=\left(a_{i 1}, \ldots, a_{i N}\right)$ is the $i$ th row element of matrix $\mathscr{A}$. Then the closed-loop system is

$$
\begin{aligned}
\mathrm{d} X(t)= & -b(t)\left(\mathscr{L} \otimes I_{n}\right) X\left(t_{k}\right) \mathrm{d} t \\
& +b(t)\left(J \otimes I_{n}\right) \mathrm{d} \Lambda(t), \\
& t \in\left[t_{k}, t_{k+1}\right), k=0,1, \cdots
\end{aligned}
$$

where $\Lambda(t)=\left(\Lambda_{1}^{T}(t), \ldots, \Lambda_{N}^{T}(t)\right)^{T}$ and $\Lambda_{i}(t)=\left(\Lambda_{1 i}^{T}(t), \ldots\right.$, $\left.\Lambda_{N i}^{T}(t)\right)^{T}, i=1, \ldots, N$. For $i, j=1, \ldots, N, \int_{0}^{t} \varphi_{j i}(s) d s=$ $\Lambda_{j i}(t)$ is $n$ dimensional standard Brownian motion. Let $e(t)=$ $\left(e_{1}(t), \ldots, e_{N}(t)\right)^{T}$ be the measurement error, where $e_{i}(t)=$ $x_{i}\left(t_{k}\right)-x_{i}(t), t \in\left[t_{k}, t_{k+1}\right), k=0,1, \cdots$. Then (3) is changed to

$$
\begin{aligned}
\mathrm{d} X(t)= & -b(t)\left(\mathscr{L} \otimes I_{n}\right)(X(t)+e(t)) \mathrm{d} t \\
& +b(t)\left(J \otimes I_{n}\right) \mathrm{d} \Lambda(t), \\
& t \in\left[t_{k}, t_{k+1}\right), k=0,1, \cdots
\end{aligned}
$$

We present the following definition of event-triggered bipartite consensus for the stochastic system.

Definition 3. Let $\mathcal{U}=\left\{u_{i}, i=1, \ldots, N\right\}$ be an eventtriggered protocol. If for any given $X(0) \in R^{n N}$, there exist $g=\left(g_{1}, \ldots, g_{N}\right)^{T} \in R^{N}, g_{i} \in\{ \pm 1\}, i=1, \ldots, N$ and $n$ dimensional random vector $\nu^{*}$,

$$
\lim _{t \rightarrow \infty} E\left\|X(t)-g \otimes v^{*}\right\|^{2}=0,
$$

where $E\left\|\nu^{*}\right\|^{2}<\infty, E \nu^{*}$ is dependent on communication relations among agents and $X(0)$, which is deterministic. 
Then, event-triggered protocol $\mathcal{U}$ is called a mean square bipartite consensus protocol.

We introduce the event-triggered condition

$$
\|e(t)\| \leq c_{1} e^{-\alpha t},
$$

where $c_{1}>0,0<\alpha<\min _{\lambda(\mathscr{L}) \neq 0}\{\operatorname{Re}(\lambda(\mathscr{L}))\}$. When the measurement error $\|e(t)\|$ is over the threshold, the controller is triggered and updates itself.

To analyze the closed-loop system in (4), we make the following assumptions:

$$
\begin{aligned}
& \left(\mathbf{Q}_{1}\right) \mathscr{G}=(\mathscr{V}, \mathscr{E}, \mathscr{A}) \text { is structurally balanced. } \\
& \left(\mathbf{Q}_{2}\right) \mathscr{G}=(\mathscr{V}, \mathscr{E}, \mathscr{A}) \text { contains a spanning tree. } \\
& \left(\mathbf{Q}_{3}\right) \int_{0}^{\infty} b(s) \mathrm{d} s=\infty . \\
& \left(\mathbf{Q}_{4}\right) \int_{0}^{\infty} b^{2}(s) \mathrm{d} s<\infty .
\end{aligned}
$$

The following lemma plays an important role in the following section.

Lemma 4 (see [16]). Given linear time-varying system

$$
\frac{d Y_{l}}{d t}=-b(t) F_{l}^{\lambda} Y_{l}, \quad l \in \mathbb{N}, \lambda \in \mathbb{C}, t \geq t_{0} \geq 0,
$$

where $Y_{l}=\left(y_{l 1}, \ldots, y_{l R_{l}}\right)^{T} \in R^{R_{l}}$ and $F_{l}^{\lambda}$ is the $R_{l} \times R_{l}$ dimensional Jordan block, which $\lambda$ is the diagonal element. Then the state transition matrix of $(7)$ is $\Psi_{F_{l}^{\lambda}}\left(t, t_{0}\right)=e^{-\int_{t_{0}}^{t} b(s) d s F_{l}^{\lambda}}$. In addition, we can obtain $\lim _{t \rightarrow \infty} \Psi_{F_{l}^{\lambda}}\left(t, t_{0}\right)=0$ if $\int_{0}^{\infty} b(s) d s=$ $\infty$ and $\operatorname{Re}(\lambda)>0$.

Lemma 5. If the event-triggered protocol (2) is a mean square bipartite consensus protocol, then $\exists g=\left(g_{1}, \ldots, g_{N}\right)^{T} \in R^{N}$, $g_{i} \in\{ \pm 1\}, i=1, \ldots, N$, and $\theta=\left(\theta_{1}, \ldots, \theta_{N}\right)^{T} \in R^{N}$, such that $\lim _{t \rightarrow \infty} \Psi(t, 0)=g \theta^{T} \otimes I_{n}$, where $\Psi(t, 0)$ is the state transition matrix of (4).

Proof. From the above condition, Definition 3 implies that for any given initial state $X(0)$, there exist a vector $g$ and a random vector $v^{*}$ so that $\lim _{t \rightarrow \infty} E\left\|X(t)-g \otimes v^{*}\right\|^{2}=0$. Obviously,

$$
\begin{aligned}
X(t)= & \Psi(t, 0) X(0) \\
& -\int_{0}^{t} b(s) \Psi(t, s)\left(\mathscr{L} \otimes I_{n}\right) e(s) \mathrm{d} s \\
& +\int_{0}^{t} b(s) \Psi(t, s)\left(J \otimes I_{n}\right) \mathrm{d} \Lambda(s) .
\end{aligned}
$$

Without loss of generality, we assume $\int_{0}^{t} b(s) \Psi(t, s)(\mathscr{L} \otimes$ $\left.I_{n}\right) e(s) \mathrm{d} s$ and $\int_{0}^{t} b(s) \Psi(t, s)\left(J \otimes I_{n}\right) \mathrm{d} \Lambda(s)$ converge to $Y^{*}$ and $Z^{*}$ in mean square sense, respectively. Then,

$$
\begin{aligned}
g \otimes E \nu^{*} & =\lim _{t \longrightarrow \infty} E X(t) \\
& =\left(\Psi_{\infty} \otimes I_{n}\right) X(0)-E Y^{*}+E Z^{*},
\end{aligned}
$$

where $\lim _{t \rightarrow \infty} \Psi(t, 0)=\Psi_{\infty} \otimes I_{n}$. According to Definition 3 and the arbitrariness of $X(0)$, one obtains $\left(\Psi_{\infty} \otimes I_{n}\right) X(0)=$ $g \otimes E d^{*}$, where $E d^{*} \in R^{N}$.

Let $\Psi_{\infty}=\left(\varrho_{1}, \ldots, \varrho_{N}\right)$. Then, all elements of $\varrho_{i}$ have the same absolute value. The same applies for $\sum_{j=1}^{N}\left(\varrho_{j} \otimes \tau_{j}\right)$, where $\tau_{j} \in R^{n}, j=1, \ldots, N$. If $\Psi_{\infty}=0$, then by making $\theta=0$, Lemma 5 holds. If $\Psi_{\infty}$ has at least one nonzero column, without loss of generality, we assume $\varrho_{1} \neq 0$. Then $\varrho_{1}=$ $\theta_{1} g$. Without loss of generality, we assume $\theta_{1}>0$. For any $\alpha, \beta \in R^{n}, a, b \in R, a, b \neq 0, a \alpha+b \beta \neq \pm(a \alpha-b \beta)$. If $\varrho_{j} \neq 0$ for some $j \neq 1$, then all $n$ dimensional components of $\varrho_{j} \otimes \alpha-\varrho_{1} \otimes \beta$ have the same modulus if and only if $\varrho_{j}=\theta_{j} g, \theta_{j}>0$. If $\varrho_{j}=0$, we have $\varrho_{j}=\theta_{j} g$ by taking $\theta_{j}=0$. Then $\Psi_{\infty}=\left(\varrho_{1}, \ldots, \varrho_{N}\right)=\left(\theta_{1} g, \ldots, \theta_{N} g\right)=g\left(\theta_{1}, \ldots, \theta_{n}\right)$. In addition, $\lim _{t \rightarrow \infty} \Psi(t, 0)=\Psi_{\infty} \otimes I_{N}$, so $\lim _{t \rightarrow \infty} \Psi(t, 0)=$ $g\left(\theta_{1}, \theta_{2}, \ldots, \theta_{N}\right) \otimes I_{n}=g \theta^{T} \otimes I_{n}$.

Lemma 6. If $\left(\mathbf{Q}_{\mathbf{1}}\right)-\left(\mathbf{Q}_{\mathbf{4}}\right)$ hold, then for any given initial state $X(0)$, there is a random vector $X^{*}$ such that $X(t)$ converges to $X^{*}$ in mean square sense, i.e., $\lim _{t \rightarrow \infty} E\left\|X(t)-X^{*}\right\|^{2}=0$.

Proof. If $\left(\mathbf{Q}_{\mathbf{1}}\right)$ and $\left(\mathbf{Q}_{2}\right)$ hold, then Laplacian $\mathscr{L}$ has exactly one zero eigenvalue and all nonzero eigenvalues have positive real parts by Lemma 1 . Thus, there exists an invertible matrix $D$, such that

$$
D^{-1} \mathscr{L} D=F=\operatorname{diag}\left(0, F_{2}, \ldots, F_{\gamma}\right)
$$

where $F_{i}(i=2, \ldots, \gamma)$ is the $R_{i} \times R_{i}$ dimensional Jordan block, which $\lambda_{i}$ is the diagonal element, and $R_{2}+\cdots+R_{\gamma}=N-$ 1. Obviously, $\lambda_{2}, \ldots, \lambda_{\gamma}$ are eigenvalues of $\mathscr{L}$ and $\operatorname{Re}\left(\lambda_{i}\right)>$ $0, i=2, \ldots, \gamma$.

Since $\Psi\left(t, t_{0}\right)\left(t_{0} \geq 0\right)$ is the state transition matrix of (4), $\Psi\left(t, t_{0}\right)=e^{-\int_{t_{0}}^{t} b(s) \mathrm{d} s \mathscr{L}} \otimes I_{n}$. From Lemma 4 ,

$$
\begin{aligned}
& \Psi\left(t, t_{0}\right)=\left(D \otimes I_{n}\right) \\
& \cdot \operatorname{diag}\left(I_{n}, \Psi_{F_{2}^{\lambda_{2}}}\left(t, t_{0}\right) \otimes I_{n}, \ldots, \Psi_{F_{\gamma}^{\lambda_{\gamma}}}\left(t, t_{0}\right) \otimes I_{n}\right) \\
& \quad \cdot\left(D^{-1} \otimes I_{n}\right) .
\end{aligned}
$$

Combining this with $\left(\mathbf{Q}_{\mathbf{3}}\right)$, one has

$$
\lim _{t \longrightarrow \infty} \Psi\left(t, t_{0}\right)=\left[D \operatorname{diag}(1,0,0, \ldots, 0) D^{-1}\right] \otimes I_{n}
$$

Thus, there exists $T>0$ so that for any $t \geq t_{0}>0$,

$$
\max \left(\left\|\Psi\left(t, t_{0}\right)\right\|_{1},\left\|\Psi\left(t, t_{0}\right)\right\|_{\infty}\right) \leq T<\infty .
$$

By Itô formula, the solution of (4) is given by

$$
\begin{aligned}
X(t)= & \Psi(t, 0) X(0) \\
& -\int_{0}^{t} b(s) \Psi(t, s)\left(\mathscr{L} \otimes I_{n}\right) e(s) \mathrm{d} s \\
& +\int_{0}^{t} b(s) \Psi(t, s)\left(J \otimes I_{n}\right) \mathrm{d} \Lambda(s)
\end{aligned}
$$


By $\left(\mathbf{Q}_{4}\right)$, one obtains that, $\forall \varepsilon>0, \exists \Gamma_{0}>0, \int_{\Gamma_{0}}^{\infty} b^{2}(s) \mathrm{d} s<$ $\varepsilon$. By (12), $\exists \Gamma_{1}>\Gamma_{0}$, such that $\max \left(\left\|\Psi\left(t_{2}, t_{3}\right)-\Psi\left(t_{1}, t_{3}\right)\right\|_{1}\right.$, $\left.\left\|\Psi\left(t_{2}, t_{3}\right)-\Psi\left(t_{1}, t_{3}\right)\right\|_{\infty}\right)<\varepsilon, t_{2} \geq t_{1}>\Gamma_{1}, \forall t_{3} \in\left[0, \Gamma_{0}\right]$.

Let $X_{2}(t)=\int_{0}^{t} b(s) \Psi(t, s)\left(\mathscr{L} \otimes I_{n}\right) e(s) \mathrm{d} s$, then by (10) and (11), one has

$$
\begin{aligned}
& X_{2}(t)=\int_{0}^{t} b(s)\left(D \otimes I_{n}\right) \\
& \cdot \operatorname{diag}\left(I_{n}, \Psi_{F_{2}^{\lambda_{2}}}(t, s) \otimes I_{n}, \ldots, \Psi_{F_{\gamma}^{\lambda_{\gamma}}}(t, s) \otimes I_{n}\right) \\
& \cdot\left(D^{-1} \mathscr{L} \otimes I_{n}\right) e(s) \mathrm{d} s .
\end{aligned}
$$

By (6), (10), and direct calculation, one has $\left(D^{-1} \mathscr{L} \otimes I_{n}\right) e(s)=$ $\left(0, \mathscr{D}_{2}^{T}(s), \ldots, \mathscr{D}_{N}^{T}(s)\right)^{T}$, where $\mathscr{D}_{i}(s)(i=2, \ldots, N)$ is the linear combination of $e_{1}(s), \ldots, e_{N}(s)$. By L'Hospital and direct calculation, one obtains

$$
\begin{array}{r}
\lim _{t \rightarrow \infty} \int_{0}^{t} b(s) e^{-\lambda_{i} \int_{s}^{t} b(\tau) \mathrm{d} \tau}\left(\int_{s}^{t} b(\tau) \mathrm{d} \tau\right)^{m} e^{-\alpha s} \mathrm{~d} s=0, \\
m=0,1, \ldots, R_{i}-1 ; i=2, \ldots, \gamma .
\end{array}
$$

Noticing that $\Psi_{F_{i}^{\lambda_{i}}}(t, s)=\sum_{d=0}^{\infty}\left(\left(-\int_{s}^{t} b(\tau) \mathrm{d} \tau\right)^{d}\left(F_{i}^{\lambda_{i}}\right)^{d} / d !\right)$, one has $\lim _{t \rightarrow \infty} X_{2}(t)=0$.

Let $X_{3}(t)=\int_{0}^{t} b(s) \Psi(t, s)\left(J \otimes I_{n}\right) \mathrm{d} \Lambda(s)$, then

$$
\begin{aligned}
X_{3}\left(t_{2}\right)-X_{3}\left(t_{1}\right) & \\
= & \int_{0}^{t_{2}} b(s)\left[\Psi\left(t_{2}, s\right)-\Psi\left(t_{1}, s\right)\right]\left(J \otimes I_{n}\right) \mathrm{d} \Lambda(s) \\
& \quad+\int_{t_{1}}^{t_{2}} b(s) \Psi\left(t_{1}, s\right)\left(J \otimes I_{n}\right) \mathrm{d} \Lambda(s) \triangleq X_{31}+X_{32} .
\end{aligned}
$$

Therefore $E\left\|X_{3}\left(t_{2}\right)-X_{3}\left(t_{1}\right)\right\|^{2} \leq 2 E\left\|X_{31}\right\|^{2}+2 E\left\|X_{32}\right\|^{2}$. It is easy to obtain

$$
\begin{aligned}
E \| & X_{31} \|^{2} \\
& =\int_{0}^{t_{2}} b^{2}(s)\left\|\left[\Psi\left(t_{2}, s\right)-\Psi\left(t_{1}, s\right)\right]\left(J \otimes I_{n}\right)\right\|_{F}^{2} \mathrm{~d} s .
\end{aligned}
$$

Noting

$$
\begin{gathered}
\int_{0}^{T_{0}} b^{2}(s)\left\|\left[\Psi\left(t_{2}, s\right)-\Psi\left(t_{1}, s\right)\right]\left(J \otimes I_{n}\right)\right\|_{F}^{2} \mathrm{~d} s \\
\leq n N\left\|\left(J \otimes I_{n}\right)\right\|_{1}^{2} \int_{0}^{\infty} b^{2}(s) \mathrm{d} s \triangleq M_{4} \varepsilon^{2}
\end{gathered}
$$

and

$$
\begin{aligned}
& \int_{\Gamma_{0}}^{t_{2}} b^{2}(s)\left\|\left[\Psi\left(t_{2}, s\right)-\Psi\left(t_{1}, s\right)\right]\left(J \otimes I_{n}\right)\right\|_{F}^{2} \mathrm{~d} s \\
& \quad \leq 4 n N L^{2}\left\|\left(J \otimes I_{n}\right)\right\|_{1}^{2} \varepsilon \triangleq M_{5} \varepsilon,
\end{aligned}
$$

one has $E\left\|X_{31}\right\|^{2} \leq M_{4} \varepsilon^{2}+M_{5} \varepsilon$. Similarly, one obtains

$$
\begin{aligned}
E\left\|X_{32}\right\|^{2} & =\int_{t_{1}}^{t_{2}} b^{2}(s)\left\|\Psi\left(t_{1}, s\right)\left(J \otimes I_{n}\right)\right\|_{F}^{2} \mathrm{~d} s \\
& \leq n N L^{2}\left\|\left(J \otimes I_{n}\right)\right\|_{1}^{2} \varepsilon \triangleq \frac{M_{5}}{4} \varepsilon .
\end{aligned}
$$

So $E\left\|X_{3}\left(t_{2}\right)-X_{3}\left(t_{1}\right)\right\|^{2} \leq 2 M_{4} \varepsilon^{2}+(5 / 2) M_{5} \varepsilon$. By Cauchy criterion and the arbitrariness of $\varepsilon$, there exists $X_{3}^{*}$ such that $X_{3}(t)$ converges to $X_{3}^{*}$ in mean square sense. So there exists $X^{*}$ such that $X(t)$ converges to $X^{*}$ in mean square sense. By (12), $X^{*}=\left[D \operatorname{diag}(1,0, \ldots, 0) D^{-1} \otimes I_{n}\right] X(0)+X_{3}^{*}$.

\section{Main Results}

In this section, we give necessary and sufficient conditions for the proposed event-triggered protocols to guarantee a mean square bipartite consensus.

Theorem 7. The event-triggered protocol in (2) is a mean square bipartite consensus protocol for the system in (1) if and only if $\left(\mathbf{Q}_{1}\right)-\left(\mathbf{Q}_{4}\right)$ hold.

\section{Proof (sufficiency).}

(S.1) Construct a Bipartition for the MAS. By $\left(\mathbf{Q}_{3}\right), \mathscr{V}$ can be decomposed into two disjoint subsets $\mathscr{V}_{v_{1}}, \mathscr{V}_{v_{2}}, \mathscr{V}_{v_{1}} \cup \mathscr{V}_{v_{2}}=$ $\mathscr{V}, \mathscr{V}_{v_{1}} \cap \mathscr{V}_{v_{2}}=\varnothing$, and $a_{s t} \leq 0$ for $s \in \mathscr{V}_{f}, t \in \mathscr{V}_{g}, f \neq$ $g, f, g \in\left\{v_{1}, v_{2}\right\}$, and $a_{s t} \geq 0$ for $s, t \in \mathscr{V}_{p}, p \in\left\{v_{1}, v_{2}\right\}$. Without loss of generality, we assume $\mathscr{V}_{v_{1}}=\{1, \ldots, m\}$, $\mathscr{V}_{v_{2}}=\{m+1, \ldots, N\}$. Let $g_{i}=1$ for $i \in \mathscr{V}_{v_{1}}$ and $g_{j}=-1$ for $j \in \mathscr{V}_{v_{2}}$. By definition, one has $\mathscr{L} g=0$, where $g=$ $\left(g_{1}, \ldots, g_{N}\right)^{T}$.

(S.2) Prove $\lim _{t \rightarrow \infty} E\left\|X(t)-g \otimes v^{*}\right\|^{2}=0$. From Lemma 6, $\exists X^{*}, \lim _{t \rightarrow \infty} E\left\|X(t)-X^{*}\right\|^{2}=0$. Without loss of generality, we assume $\lim _{t \rightarrow \infty} E\left\|x_{1}(t)-v^{*}\right\|^{2}=0$. Next, we will prove $\lim _{t \rightarrow \infty} E\left\|x_{i}(t)-g_{i} v^{*}\right\|^{2}=0, i=2, \ldots, N$.

Let $\phi(t)=\left(\phi_{2}^{T}(t), \ldots, \phi_{N}^{T}(t)\right)^{T}$, where $\phi_{i}(t)=x_{i}(t)-$ $g_{i} x_{1}(t), i=2, \ldots, N$. Now we prove that $\lim _{t \rightarrow \infty} E\|\phi(t)\|^{2}=$ 0 . For this purpose, We assume $\widetilde{\phi}(t) \triangleq\left(Q \otimes I_{n}\right) X(t)$, where

$$
\begin{aligned}
Q & \triangleq\left(\begin{array}{c|c|c}
\frac{1}{N} & \frac{1}{N} \cdots \frac{1}{N} & -\frac{1}{N} \cdots-\frac{1}{N} \\
-\mathbf{1}_{m-1} & I_{m-1} & 0 \\
-\mathbf{1}_{N-m} & 0 & -I_{N-m}
\end{array}\right) \\
& =\left(\begin{array}{c}
\frac{1}{N} g^{T} \\
Q_{2}
\end{array}\right) .
\end{aligned}
$$

Then $\widetilde{\phi}(t)=\left(\chi^{T}(t), \phi^{T}(t)\right)^{T}$, where $\chi(t) \triangleq(1 / N)\left(g^{T} \otimes\right.$ $\left.I_{n}\right) X(t)$. Since

$$
\mathrm{QLQ}^{-1}=\left(\begin{array}{cc}
0 & \varpi^{T} \\
0 & L_{2}
\end{array}\right)
$$


by (4), one has

$$
\begin{aligned}
d \phi(t)= & -b(t)\left(L_{2} \otimes I_{n}\right) \phi(t) \mathrm{d} t \\
& -b(t)\left(Q_{2} L \otimes I_{n}\right) e(t) \mathrm{d} t \\
& +b(t)\left(Q_{2} J \otimes I_{n}\right) \mathrm{d} \Lambda(t) .
\end{aligned}
$$

By (23), $S^{-1} L_{2} S=\operatorname{diag}\left(F_{2}, \ldots, F_{\gamma}\right)$, where $S$ is invertible and $F_{2}, \ldots, F_{\gamma}$ are given in (10). The state transition matrix of the system in (24) is

$$
\begin{aligned}
\Psi_{2}\left(t, t_{0}\right)=\left(S \otimes I_{n}\right) \\
\cdot\left[\operatorname{diag}\left(\Psi_{F_{2}^{\lambda_{2}}}\left(t, t_{0}\right) \otimes I_{n}, \ldots, \Psi_{F_{\gamma}^{\lambda_{\gamma}}}\left(t, t_{0}\right) \otimes I_{n}\right)\right] \\
\cdot\left(S^{-1} \otimes I_{n}\right),
\end{aligned}
$$

where $\Psi_{F_{q} \lambda_{q}}\left(t, t_{0}\right), q=2, \ldots, \gamma$ are defined as in Lemma 4 . Hence, $\lim _{t \rightarrow \infty} \Psi_{2}\left(t, t_{0}\right)=0$, i.e., $\forall \varepsilon>0, \exists \Gamma_{2}>\Gamma_{1}$, such that $\left\|\Psi_{2}\left(t, t_{0}\right)\right\|<\varepsilon, \forall t>\Gamma_{2}$. Furthermore, $\exists T_{L_{2}}>0$, such that, $\forall t>t_{0} \geq 0, \max \left(\left\|\Psi_{2}\left(t, t_{0}\right)\right\|_{1},\left\|\Psi_{2}\left(t, t_{0}\right)\right\|_{\infty}\right) \leq T_{L_{2}}<\infty$.

By Itô formula, it can be seen that the state of the system in (24) can be described as

$$
\begin{aligned}
\phi(t)= & \Psi_{2}(t, 0) \phi(0) \\
& -\int_{0}^{t} b(s) \Psi_{2}(t, s)\left(Q_{2} L \otimes I_{n}\right) e(s) \mathrm{d} s \\
& +\int_{0}^{t} b(s) \Psi_{2}(t, s)\left(Q_{2} J \otimes I_{n}\right) \mathrm{d} \Lambda(s) .
\end{aligned}
$$

Therefore,

$$
\begin{gathered}
E\|\phi(t)\|^{2}=\left\|\Psi_{2}(t, 0) \phi(0)\right\|^{2}-2 \phi^{T}(0) \Psi_{2}^{T}(t, 0) \\
\cdot \int_{0}^{t} b(s) \Psi_{2}(t, s)\left(Q_{2} L \otimes I_{n}\right) e(s) \mathrm{d} s \\
\quad+\left\|\int_{0}^{t} b(s) \Psi_{2}(t, s)\left(Q_{2} L \otimes I_{n}\right) e(s) \mathrm{d} s\right\|^{2} \\
\quad+\int_{0}^{t} b^{2}(s)\left\|\Psi_{2}(t, s)\left(Q_{2} J \otimes I_{n}\right)\right\|_{F}^{2} \mathrm{~d} s,
\end{gathered}
$$

and hence,

$$
\begin{aligned}
E\|\phi(t)\|^{2} & \\
\leq & 2\left\|\Psi_{2}(t, 0) \phi(0)\right\|^{2} \\
& +2\left\|\int_{0}^{t} b(s) \Psi_{2}(t, s)\left(Q_{2} L \otimes I_{n}\right) e(s) \mathrm{d} s\right\|^{2} \\
& +\int_{0}^{t} b^{2}(s)\left\|\Psi_{2}(t, s)\left(Q_{2} J \otimes I_{n}\right)\right\|_{F}^{2} \mathrm{~d} s .
\end{aligned}
$$

Since

$$
\begin{aligned}
& \left\|\int_{0}^{t} b(s) \Psi_{2}(t, s)\left(Q_{2} L \otimes I_{n}\right) e(s) \mathrm{d} s\right\|^{2} \\
& \leq \int_{0}^{t} b^{2}(s)\left\|\Psi_{2}(t, s)\right\|^{2} \mathrm{~d} s \int_{0}^{t}\left\|\left(Q_{2} L \otimes I_{n}\right)\right\|^{2}\|e(s)\|^{2} \mathrm{~d} s
\end{aligned}
$$

and

$$
\begin{aligned}
& \int_{0}^{t}\left\|\left(Q_{2} L \otimes I_{n}\right)\right\|^{2}\|e(s)\|^{2} \mathrm{~d} s \\
& \quad \leq\left\|\left(Q_{2} L \otimes I_{n}\right)\right\|^{2} \int_{0}^{t} c_{1}^{2} e^{-2 \alpha s} \mathrm{~d} s \leq \frac{c_{1}^{2}}{2 \alpha}\left\|\left(Q_{2} L \otimes I_{n}\right)\right\|^{2},
\end{aligned}
$$

there exists $\beta_{3}>0$ such that $\int_{0}^{t}\left\|\left(Q_{2} L \otimes I_{n}\right)\right\|^{2}\|e(s)\|^{2} \mathrm{~d} s \leq \beta_{3}$. Then

$$
\begin{gathered}
\left\|\int_{0}^{t} b(s) \Psi_{2}(t, s)\left(Q_{2} L \otimes I_{n}\right) e(s) \mathrm{d} s\right\|^{2} \\
\leqslant \beta_{3} \int_{0}^{\Gamma_{0}} b^{2}(s)\left\|\Psi_{2}(t, s)\right\|^{2} \mathrm{~d} s \\
\quad+\beta_{3} \int_{\Gamma_{0}}^{t} b^{2}(s)\left\|\Psi_{2}(t, s)\right\|^{2} \mathrm{~d} s .
\end{gathered}
$$

Since $\int_{0}^{\Gamma_{0}} b^{2}(s)\left\|\Psi_{2}(t, s)\right\|^{2} \mathrm{~d} s=\left\|\Psi_{2}(t, \eta)\right\|^{2} \int_{0}^{\Gamma_{0}} b^{2}(s) \mathrm{d} s \leq \| \Psi_{2}(t$, $\eta) \|^{2} \int_{0}^{\infty} b^{2}(s) \mathrm{d} s$, where $\eta \in\left(0, \Gamma_{0}\right)$ and $\int_{\Gamma_{0}}^{\infty} b^{2}(s) \mathrm{d} s<\varepsilon$, one has $\int_{\Gamma_{0}}^{t} b^{2}(s)\left\|\Psi_{2}(t, s)\right\|^{2} \mathrm{~d} s \leq T_{L_{2}}^{2} \varepsilon \triangleq M_{6} \varepsilon$. Therefore, $\forall t>T_{2}$

$$
\begin{aligned}
& \left\|\int_{0}^{t} b(s) \Psi_{2}(t, s)\left(Q_{2} L \otimes I_{n}\right) e(s) \mathrm{d} s\right\|^{2} \\
& \quad \leq \beta_{3}\left\|\Psi_{2}(t, \eta)\right\|^{2} \int_{0}^{\infty} b^{2}(s) \mathrm{d} s+M_{6} \beta_{3} \varepsilon \\
& \quad \leq \varepsilon^{2} \beta_{3} \int_{0}^{\infty} b^{2}(s) \mathrm{d} s+M_{6} \beta_{3} \varepsilon .
\end{aligned}
$$

From $\int_{\Gamma_{0}}^{\infty} b^{2}(s) d s<\varepsilon$, one gets

$$
\begin{aligned}
& \int_{\Gamma_{0}}^{t} b^{2}(s)\left\|\Psi_{2}(t, s)\left(Q_{2} J \otimes I_{n}\right)\right\|_{F}^{2} \mathrm{~d} s \\
& \quad<\left.n N T_{L_{2}}^{2}\left\|\left(Q_{2} J \otimes I_{n}\right)\right\|\right|_{1} ^{2} \varepsilon \triangleq M_{7} \varepsilon .
\end{aligned}
$$

Combining this with

$$
\begin{aligned}
& \int_{0}^{\Gamma_{0}} b^{2}(s)\left\|\Psi_{2}(t, s)\left(Q_{2} J \otimes I_{n}\right)\right\|_{F}^{2} \mathrm{~d} s \\
& \leq n N\left\|\Psi_{2}(t, \xi)\right\|_{1}^{2}\left\|\left(Q_{2} J \otimes I_{n}\right)\right\|_{1}^{2} \int_{0}^{\infty} b^{2}(s) \mathrm{d} s, \\
& \xi \in\left(0, \Gamma_{0}\right),
\end{aligned}
$$

one has

$$
\begin{aligned}
E\|\phi(t)\|^{2} \leq & 2\|\phi(0)\|^{2} \varepsilon^{2}+2 \beta_{3} \varepsilon^{2} \int_{0}^{\infty} b^{2}(s) \mathrm{d} s \\
& +n N \varepsilon^{2}\left\|\left(Q_{2} J \otimes I_{n}\right)\right\|_{1}^{2} \int_{0}^{\infty} b^{2}(s) \mathrm{d} s \\
& +2 M_{6} \beta_{3} \varepsilon+M_{7} \varepsilon .
\end{aligned}
$$


By the arbitrariness of $\varepsilon$, one gets $\lim _{t \rightarrow \infty} E\|\phi(t)\|^{2}=0$. Hence, $\lim _{t \rightarrow \infty} E\left\|X(t)-g \otimes v^{*}\right\|^{2}=0$.

(S.3) Analyze the Statistical Characteristics of $v^{*}$. By Lemma 6, $g \otimes v^{*}=X^{*}=\left[D \operatorname{diag}(1,0,0, \ldots, 0) D^{-1} \otimes I_{n}\right] X(0)+X_{3}^{*}$. So $g \otimes E v^{*}=E X^{*}=\left[D \operatorname{diag}(1,0,0, \ldots, 0) D^{-1} \otimes I_{n}\right] X(0)$.

We assume $m_{r}, m_{l}^{T}$ represent the first column of $D$ and the first row of $D^{-1}$, respectively. Then, $g \otimes E v^{*}=\left(m_{r} m_{l}^{T} \otimes\right.$ $\left.I_{n}\right) X(0)$. Since $D^{-1} \mathscr{L} D=F, \mathscr{L} D=D F$, and $D^{-1} \mathscr{L}=F D^{-1}$, $\mathscr{L} m_{r}=0$ and $m_{l}^{T} \mathscr{L}=0$. By (S.1), $\mathscr{L} g=0$. Therefore, $m_{r}=$ $\kappa g(\kappa \in R$ and $\kappa \neq 0)$ and $g \otimes E v^{*}=g \otimes\left[\kappa\left(m_{l}^{T} \otimes I_{n}\right) X(0)\right]$. Then $E v^{*}=\kappa\left(m_{l}^{T} \otimes I_{n}\right) X(0)$. Clearly, $m_{l}$ is concerned with communication topology. Thus, $E v^{*}$ is determined by $X(0)$ and communication topology of MASs.

It is easy to obtain that $\Psi($,$) is uniformly bounded.$ Therefore, $\forall \varepsilon>0, \exists \Gamma_{3}>\Gamma_{2}$,

$$
\begin{aligned}
& \int_{\Gamma_{3}}^{\infty} b^{2}(s)\left\|\Psi(t, s)\left(J \otimes I_{n}\right)\left(J \otimes I_{n}\right)^{T} \Psi^{T}(t, s)\right\| \mathrm{d} s<\varepsilon \\
& \int_{\Gamma_{3}}^{\infty} b^{2}(s) \|\left(m_{r} m_{l}^{T} \otimes I_{n}\right)\left(J \otimes I_{n}\right)\left(J \otimes I_{n}\right)^{T} \\
& \cdot\left(m_{r} m_{l}^{T} \otimes I_{n}\right)^{T} \| \mathrm{d} s<\varepsilon .
\end{aligned}
$$

Let $X_{4}(t)=\int_{0}^{\Gamma_{3}} b^{2}(s) \Psi(t, s)\left(J \otimes I_{n}\right)\left(J \otimes I_{n}\right)^{T} \Psi^{T}(t, s) \mathrm{d} s$. Then for any $t>\Gamma_{4}, \| X_{4}(t)-\int_{0}^{\Gamma_{3}} b^{2}(s)\left(m_{r} m_{l}^{T} \otimes I_{n}\right)(J \otimes$ $\left.I_{n}\right)\left(J \otimes I_{n}\right)^{T}\left(m_{r} m_{l}^{T} \otimes I_{n}\right)^{T} \mathrm{~d} s \|<\varepsilon$. This together with (36) leads to $\lim _{t \rightarrow \infty} \int_{0}^{t} b^{2}(s) \Psi(t, s)\left(J \otimes I_{n}\right)\left(J \otimes I_{n}\right)^{T} \Psi^{T}(t, s) \mathrm{d} s=$ $\int_{0}^{\infty} b^{2}(s)\left(m_{r} m_{l}^{T} J J^{T} m_{l} m_{r}^{T} \otimes I_{n}\right) \mathrm{d} s=\Theta\left(g g^{T} \otimes I_{n}\right)$, where $\Theta=\kappa^{2}\left(m_{l}^{T} J J^{T} m_{l}\right) \int_{0}^{\infty} b^{2}(s) \mathrm{d} s$. Combining this with $D\left(X^{*}\right)=$ $\lim _{t \rightarrow \infty} \int_{0}^{t} b^{2}(s) \times \Psi(t, s)\left(J \otimes I_{n}\right)\left(J \otimes I_{n}\right)^{T} \Psi^{T}(t, s) \mathrm{d} s$, one gets $D\left(\nu^{*}\right)=\Theta I_{n}$. Therefore, $E\left\|\nu^{*}\right\|^{2}<\infty$. By Definition 3, the sufficiency is established.

Necessity.

(B.1) Prove $\left(Q_{3}\right)$, Namely, $\int_{0}^{\infty} b(s) d s=\infty$. By contradiction, we assume that $\left(\mathbf{Q}_{3}\right)$ does not hold. Then, $\exists \bar{\epsilon}>0$, $\int_{0}^{\infty} b(s) \mathrm{d} s=\bar{\epsilon}$, and $\lim _{t \rightarrow \infty} e^{-\int_{0}^{t} b(s) \mathrm{d} s \mathscr{L}}=e^{-\bar{\epsilon} \mathscr{L}}$. Therefore, $\operatorname{rank}\left(\lim _{t \rightarrow \infty} \Psi(t, 0)\right)=\operatorname{rank}\left(e^{-\bar{\epsilon} \mathscr{L}} \otimes I_{n}\right)=n N$. However, by Lemma 5, $\lim _{t \rightarrow \infty} \Psi(t, 0)=g \theta^{T} \otimes I_{n}$ and $\operatorname{rank}\left(\lim _{t \rightarrow \infty} \Psi(t, 0)\right) \leq n$. This is a contradiction. So $\left(\mathbf{Q}_{3}\right)$ holds.

(B.2) Prove That Laplacian Matrix $\mathscr{L}$ Has Exactly One Zero Eigenvalue. By contradiction, we assume that 0 is not an eigenvalue of $\mathscr{L}$. Then all the eigenvalues of $\mathscr{L}$ have positive real part and $-\mathscr{L}$ is a Hurwitz matrix. By $\left(\mathbf{Q}_{3}\right)$ and Lemma 4 , $\lim _{t \rightarrow \infty} \Psi(t, 0)=0$. Combining this with Lemma 5, one has $g \otimes E v^{*}=E Z^{*}-E Y^{*}$. Since $E Z^{*}$ and $E Y^{*}$ are independent of $X(0), E v^{*}$ is independent of $X(0)$. This contradicts Definition 3. So 0 is an eigenvalue of $\mathscr{L}$.

Let $F_{1}^{0}$ be a Jordan block with eigenvalue 0 . Then it is 1 dimensional. Otherwise, we assume $F_{1}^{0}$ is $R_{1}$ dimensional and $R_{1}>1$. Then, by $\left(\mathbf{Q}_{3}\right)$ and the definition of matrix exponent function, one gets that $\lim _{t \rightarrow \infty} e^{-\int_{0}^{t} b(s) \mathrm{d} s F_{1}^{0}}$ does not exist, and hence, $\lim _{t \rightarrow \infty} \Psi(t, 0)$ does not exist. This contradicts Lemma 5. So $F_{1}^{0}$ is 1 dimensional.

Let algebra multiplicity of eigenvalue 0 be $w$. Then $w=1$. Otherwise, $w>1$. Take $w=2$ as an example. Since each Jordan block corresponding to eigenvalue 0 is 1 dimensional,

$$
\begin{aligned}
\Psi_{\infty} \otimes I_{n} & \triangleq \lim _{t \rightarrow \infty} \Psi(t, 0)=\lim _{t \rightarrow \infty} e^{-\int_{0}^{t} b(s) \mathrm{d} s \mathscr{L}} \otimes I_{n} \\
& =D \operatorname{diag}(1,1,0, \ldots, 0) D^{-1} \otimes I_{n} .
\end{aligned}
$$

Thus, $\operatorname{rank}\left(\Psi_{\infty}\right)=2$. This contradicts $\operatorname{rank}\left(\Psi_{\infty}\right) \leq 1$ from Lemma 6. So Laplacian $\mathscr{L}$ has exactly one zero eigenvalue.

(B.3) Prove $\left(Q_{1}\right)$ and $\left(Q_{2}\right)$. By (B.2) and $\left(\mathbf{Q}_{3}\right)$, one has (12). By Lemma 5, one gets

$$
D \operatorname{diag}(1,0,0, \ldots, 0) D^{-1}=g \theta^{T} .
$$

Noticing that $m_{r}$ is the first column of $D$, one has $\mathscr{L} m_{r}=$ 0 . By (38), one obtains $m_{r}=g \kappa^{*}$, where $\kappa^{*}=\theta^{T} m_{r} \in R$. Then, $\mathscr{L} g=0$. By the definition of $\mathscr{L}$, for any $j$, we obtain $g_{j} \sum_{k \neq j}\left|a_{j k}\right|=\sum_{k \neq j} g_{k} a_{j k}, j=1, \ldots, N$. Since $g_{j}= \pm 1$ and $g_{j}^{2}=1, j=1,2, \ldots, N, \sum_{k \neq j}\left|a_{j k}\right|=\sum_{k \neq j} g_{j} g_{k} a_{j k}$. So $g_{j} g_{k} a_{j k}=\left|a_{j k}\right| \geq 0$. Let $V_{1}=\left\{j \mid g_{j}=1\right\}$ and $V_{2}=\left\{j \mid g_{j}=\right.$ $-1\}$, then $V_{1} \cap V_{2}=\varnothing, V_{1} \cup V_{2}=V$. If $j \in \mathscr{V}_{p}, p \in\{1,2\}$, then $a_{j k} \geq 0, k \in \mathscr{V}_{p}$ or $a_{j k} \leq 0, k \in \mathscr{V}_{r}, r \neq p, r \in\{1,2\}$. By definition, $\mathscr{G}$ is structurally balanced, that is, $\left(\mathbf{Q}_{\mathbf{1}}\right)$ holds.

By (B.2) and $\left(\mathbf{Q}_{\mathbf{1}}\right)$, Lemma 1 implies that $\left(\mathbf{Q}_{2}\right)$ holds.

(B.4) Prove $\left(Q_{4}\right)$. Assume $\int_{0}^{\infty} b^{2}(s) \mathrm{d} s=\infty$. Due to the first row of $D^{-1}$ which is $m_{l}^{T}, m_{l}^{T} \mathscr{L}=0$. By (4), we obtain $\mathrm{d}\left(\left(m_{l}^{T} \otimes\right.\right.$ $\left.\left.I_{n}\right) X(t)\right)=b(t)\left(m_{l}^{T} \otimes I_{n}\right)\left(J \otimes I_{n}\right) \mathrm{d} \Lambda(t)$, i.e.

$$
\begin{aligned}
\left(m_{l}^{T} \otimes I_{n}\right) X(t) & \\
= & \left(m_{l}^{T} \otimes I_{n}\right) X(0) \\
& \quad+\left(m_{l}^{T} \otimes I_{n}\right)\left(J \otimes I_{n}\right) \int_{0}^{t} b(s) \mathrm{d} \Lambda(s) .
\end{aligned}
$$

From Definition 3, it is known that $X(t)$ converges to $g \otimes v^{*}$ in mean square sense, where $E\left\|\nu^{*}\right\|^{2}<\infty$. Thus, when $t \longrightarrow \infty,\left(m_{l}^{T} \otimes I_{n}\right)\left(J \otimes I_{n}\right) \int_{0}^{t} b(s) \mathrm{d} \Lambda(s)$ converges to a random variable $X_{m}$ in mean square sense with $E\left\|X_{m}\right\|^{2}<$ $\infty$. Then $\lim _{t \rightarrow \infty} E\left\|\left(m_{l}^{T} \otimes I_{n}\right)\left(J \otimes I_{n}\right) \int_{0}^{t} b(s) \mathrm{d} \Lambda(s)\right\|^{2}=$ $\lim _{t \rightarrow \infty} \operatorname{tr}\left(m_{l}^{T} J J^{T} m_{l} \otimes I_{n}\right) \int_{0}^{t} b^{2}(s) \mathrm{d} s=\infty$. This leads to a contradiction. So $\left(\mathbf{Q}_{\mathbf{4}}\right)$ holds.

Remark 8. From Theorem 7 it can be seen that under $\left(\mathbf{Q}_{\mathbf{1}}\right)$ $\left(\mathbf{Q}_{4}\right)$ the event-triggered protocol in (2) ensures agents converging to $v^{*}$ or $-v^{*}$ under measurement noise.

Remark 9. From Theorem 7 one sees that to guarantee the mean square bipartite consensus, $\left(\mathbf{Q}_{\mathbf{1}}\right)-\left(\mathbf{Q}_{\mathbf{2}}\right)$ are requirements for time-varying gain $b(t)$ while $\left(\mathbf{Q}_{3}\right)-\left(\mathbf{Q}_{4}\right)$ are the weakest connectivity assumptions. 


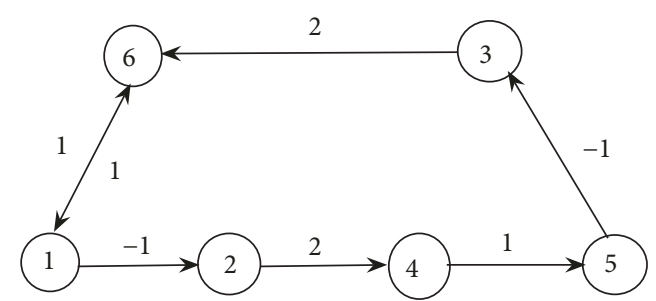

Figure 1: Communication graph $\mathscr{G}$ among the 6 agents.

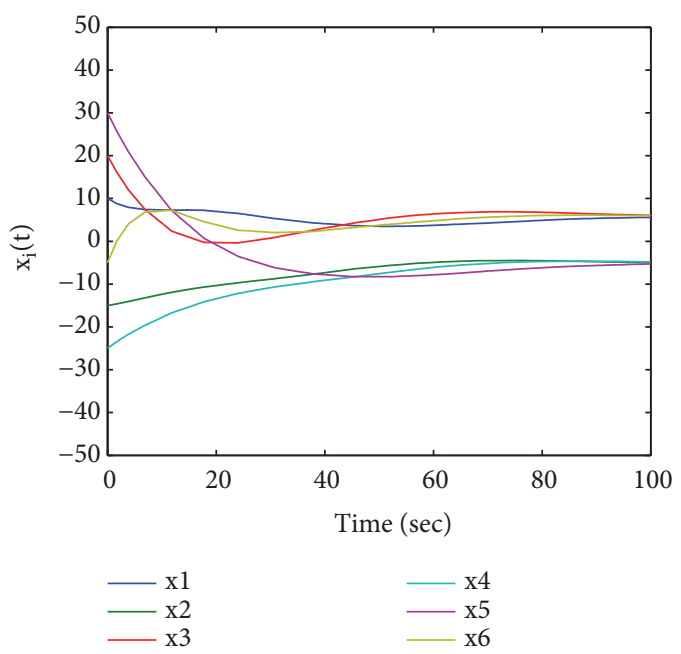

FIGURE 2: State trajectories of six agents.

\section{Numerical Simulation}

To demonstrate the developed result in the preceding, we consider an MAS of six agents, whose dynamics satisfy the system in (1). The communication graph that connects the six agents is illustrated in Figure 1. Clearly, $\mathscr{V}=\{1, \ldots, 6\}$, $\mathscr{A}=\left(a_{i j}\right), a_{16}=a_{61}=a_{54}=1, a_{21}=a_{35}=-1$, and $a_{42}=a_{63}=2$ in $\mathscr{G}=(\mathscr{V}, \mathscr{E}, \mathscr{A})$. From Figure 1 , $\mathscr{G}$ satisfies $\left(\mathbf{Q}_{\mathbf{1}}\right)$ and $\left(\mathbf{Q}_{\mathbf{2}}\right)$. Furthermore, all eigenvalues of Laplacian $\mathscr{L}$ are $\lambda_{1}=0, \lambda_{2}=0.6733+0.9192$ ر,$\lambda_{3}=0.6733-$ $0.9192 \mathrm{\jmath}, \lambda_{4}=2.0887+0.7157 \mathrm{\jmath}, \lambda_{5}=2.0887-0.7157 \mathrm{\jmath}$, and $\lambda_{6}=3.4760\left(j^{2}=-1\right)$. Obviously, $\min _{\lambda(\mathscr{L}) \neq 0}\{\operatorname{Re} \lambda(\mathscr{L})\}=$ 0.6733 . The initial state of the MAS is given by $X(0)=$ $(10,-15,20,-25,30,-5)$. Choose $b(t)=\ln (t+1) /(t+1)$. By direct calculation we know that $b(t)$ satisfies $\left(\mathbf{Q}_{3}\right)-\left(\mathbf{Q}_{\mathbf{4}}\right)$. Assume event-triggered condition (6) is satisfied by taking $c_{1}=1.2$ and $\alpha=0.6$. Applying protocol (2) to the system in (1), we get the six agents' state trajectories. As shown in Figure 2 one can see that the states of agents 1, 3, and 6 converge to 5 in mean square sense while the states of agents 2,4 , and 5 converge to -5 in mean square sense. Thus, mean square bipartite consensus is achieved with event-triggered protocol (2). On the other hand, from Figure 3 we know that the inputs are constants between the event triggering time interval. Moreover, from Figure 4, it can be seen that

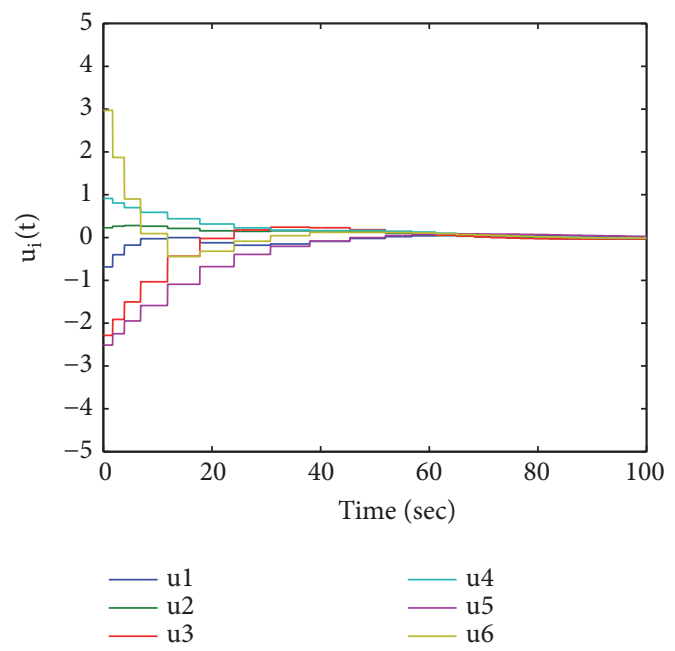

FIGURE 3: Control inputs of six agents.

the absolute value of the measurement error of each agent converges to zero. This means that the MAS does not exhibit Zeno behavior.

\section{Conclusion}

Mean square bipartite consensus problem of singleintegrator MASs is investigated in the context of eventtriggered control and measurement noise. By using timevarying gain, an event-triggered bipartite consensus protocol is proposed under measurement noise, with which the controller update frequency is reduced. With given necessary and sufficient conditions on protocol gain and communication topology, the MAS is proved to achieve event-triggered bipartite consensus. The simulation shows that the system will not show Zeno behavior.

\section{Data Availability}

The Matlab based models used to support the findings of this study are available from the corresponding author upon request.

\section{Conflicts of Interest}

The authors declare that they have no conflicts of interest.

\section{Acknowledgments}

This work was supported by the National Natural Science Foundation of China under Grants 61104136 and 61673350, Postgraduate Education Innovation Program of Shandong Province under Grant no. SDYY16088, and the Young Teacher Capability Enhancement Program for Domestic Study, Qufu Normal University. 

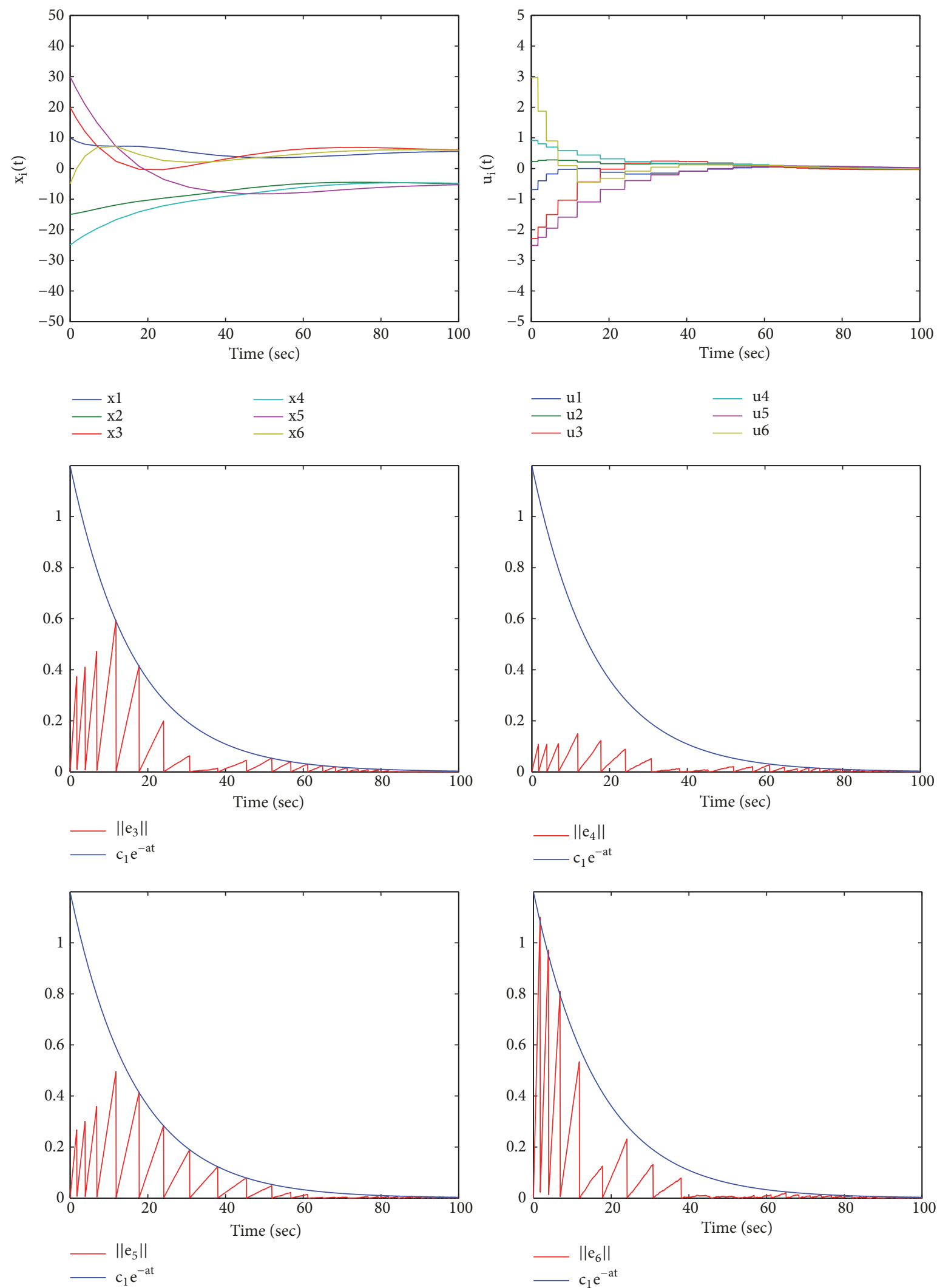

Figure 4: The evolution of error norm. 


\section{References}

[1] R. Olfati-Saber and R. M. Murray, "Consensus problems in networks of agents with switching topology and time-delays," IEEE Transactions on Automatic Control, vol. 49, no. 9, pp. 15201533, 2004.

[2] W. Ren and R. W. Beard, "Consensus seeking in multiagent systems under dynamically changing interaction topologies," IEEE Transactions on Automatic Control, vol. 50, no. 5, pp. 655661, 2005.

[3] C. Q. Ma and J. F. Zhang, "Necessary and sufficient conditions for consensusability of linear multi-agent systems," IEEE Transactions on Automatic Control, vol. 55, no. 5, pp. 1263-1268, 2010.

[4] K. You and L. Xie, "Network topology and communication data rate for consensusability of discrete-time multi-agent systems," IEEE Transactions on Automatic Control, vol. 56, no. 10, pp. 2262-2275, 2011.

[5] C. Q. Ma, T. Li, and J. F. Zhang, "Consensus control for leaderfollowing multi-agent systems with measurement noises," Journal of Systems Science and Complexity, vol. 23, no. 1, pp. 35-49, 2010.

[6] Z. Li, Z. Duan, G. Chen, and L. Huang, "Consensus of multiagent systems and synchronization of complex networks: a unified viewpoint," IEEE Transactions on Circuits and Systems I: Regular Papers, vol. 57, no. 1, pp. 213-224, 2010.

[7] C. Q. Ma and J. F. Zhang, "On formability of linear continuous multi-agent systems," Journal of Systems Science and Complexity, vol. 25, no. 1, pp. 13-29, 2012.

[8] X. Dong and G. Hu, "Time-varying formation control for general linear multi-agent systems with switching directed topologies," Automatica, vol. 73, pp. 47-55, 2016.

[9] C. Altafini, "Consensus problems on networks with antagonistic interactions," IEEE Transactions on Automatic Control, vol. 58, no. 4, pp. 935-946, 2013.

[10] J. Hu and W. X. Zheng, "Emergent collective behaviors on coopetition networks," Physics Letters A, vol. 378, no. 26-27, pp. 1787-1796, 2014.

[11] A. V. Proskurnikov, A. S. Matveev, and M. Cao, "Opinion dynamics in social networks with hostile camps: consensus vs. polarization," Institute of Electrical and Electronics Engineers Transactions on Automatic Control, vol. 61, no. 6, pp. 1524-1536, 2016.

[12] D. Meng, M. Du, and Y. Jia, "Interval bipartite consensus of networked agents associated with signed digraphs," Institute of Electrical and Electronics Engineers Transactions on Automatic Control, vol. 61, no. 12, pp. 3755-3770, 2016.

[13] C. Q. Ma and Z. Y. Qin, "Bipartite consensus on networks of agents with antagonistic interactions and measurement noises," IET Control Theory \& Applications, vol. 10, no. 17, pp. 2306-2313, 2016.

[14] J. Hu and Y. Wu, "Interventional bipartite consensus on coopetition networks with unknown dynamics," Journal of The Franklin Institute, vol. 354, no. 11, pp. 4438-4456, 2017.

[15] H. Zhang and J. Chen, "Bipartite consensus of multi-agent systems over signed graphs: state feedback and output feedback control approaches," International Journal of Robust and Nonlinear Control, vol. 27, no. 1, pp. 3-14, 2017.

[16] C. Q. Ma, Z. Y. Qin, and Y. B. Zhao, "Bipartite consensus of integrator multi-agent systems with measurement noise," IET Control Theory \& Applications, vol. 11, no. 18, pp. 3313-3320, 2017.
[17] C. Q. Ma, W. Zhao, and Y. B. Zhao, "Bipartite linear $\chi$-consensus of double-integrator multi-agent systems with measurement noise," Asian Journal of Control, vol. 20, no. 1, pp. 577-584, 2018.

[18] C. Q. Ma and L. Xie, "Necessary and sufficient conditions for leader-following bipartite consensus with measurement noise," IEEE Transactions on Systems, Man, and Cybernetics: Systems, pp. 1-6, 2018.

[19] D. V. Dimarogonas, E. Frazzoli, and K. H. Johansson, "Distributed event-triggered control for multi-agent systems," IEEE Transactions on Automatic Control, vol. 57, no. 5, pp. 1291-1297, 2012.

[20] G. S. Seyboth, D. V. Dimarogonas, and K. H. Johansson, "Event-based broadcasting for multi-agent average consensus," Automatica, vol. 49, no. 1, pp. 245-252, 2013.

[21] B. Wang, X. Meng, and T. Chen, "Event based pulse-modulated control of linear stochastic systems," Institute of Electrical and Electronics Engineers Transactions on Automatic Control, vol. 59, no. 8, pp. 2144-2150, 2014.

[22] B. Wang and M. Fu, "Comparison of periodic and event-based sampling for linear state estimation," IFAC Proceedings Volumes, vol. 47, no. 3, pp. 5508-5513, 2014.

[23] S. Liu, D. E. Quevedo, and L. Xie, "Event-triggered distributed constrained consensus," International Journal of Robust and Nonlinear Control, vol. 27, no. 16, pp. 3043-3060, 2017.

[24] E. Garcia, Y. Cao, and D. W. Casbeer, "Decentralised eventtriggered consensus of double integrator multi-agent systems with packet losses and communication delays," IET Control Theory \& Applications, vol. 10, no. 15, pp. 1835-1843, 2016.

[25] D. Yang, W. Ren, X. Liu, and W. Chen, "Decentralized eventtriggered consensus for linear multi-agent systems under general directed graphs," Automatica, vol. 69, pp. 242-249, 2016.

[26] W. Hu and L. Liu, "Cooperative output regulation of heterogeneous linear multi-agent systems by event-triggered control," IEEE Transactions on Cybernetics, vol. 47, no. 1, pp. 105-116, 2017.

[27] A. Hu, J. Cao, M. Hu, and L. Guo, "Event-triggered consensus of multi-agent systems with noises," Journal of The Franklin Institute, vol. 352, no. 9, pp. 3489-3503, 2015.

[28] Y. Zhou and J. Hu, "Event-based bipartite consensus on signed networks," in Proceedings of the 2013 IEEE 3rd Annual International Conference on Cyber Technology in Automation, Control, and Intelligent Systems (CYBER), pp. 326-330, China, May 2013.

[29] J. Zeng, F. Li, J. Qin, and W. X. Zheng, "Distributed eventtriggered bipartite consensus for multiple agents over signed graph topology," in Proceedings of the 2015 34th Chinese Control Conference (CCC), pp. 6930-6935, Hangzhou, China, July 2015. 


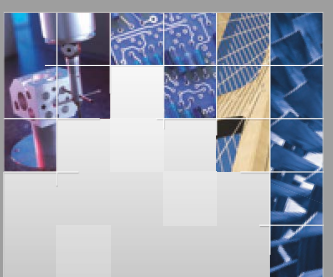

\section{Enfincering}
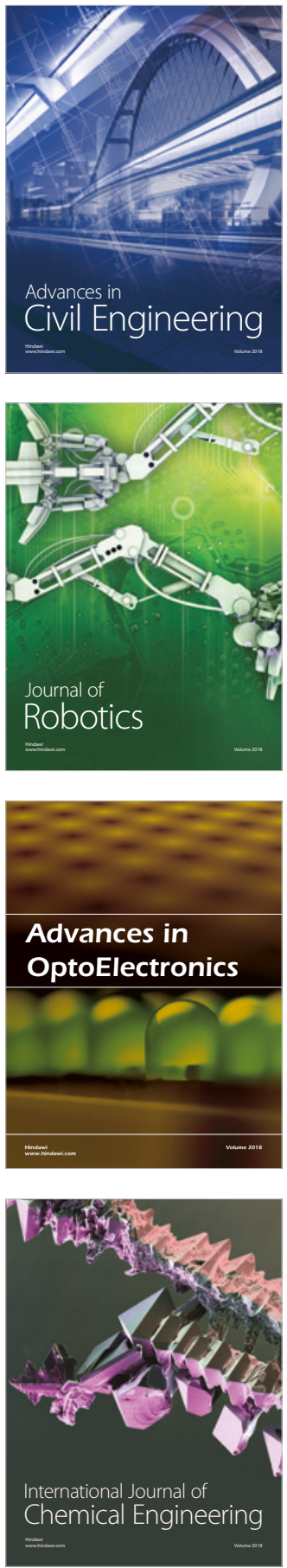

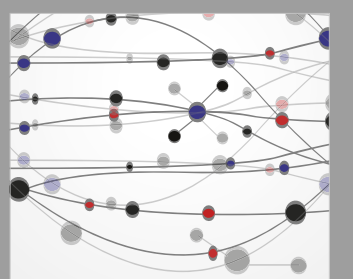

\section{Rotating \\ Machinery}

The Scientific World Journal

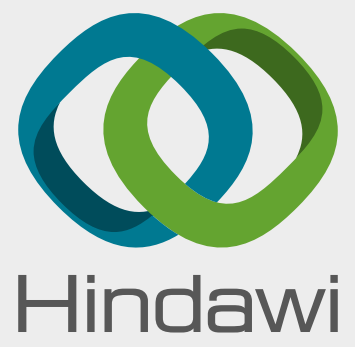

Submit your manuscripts at

www.hindawi.com
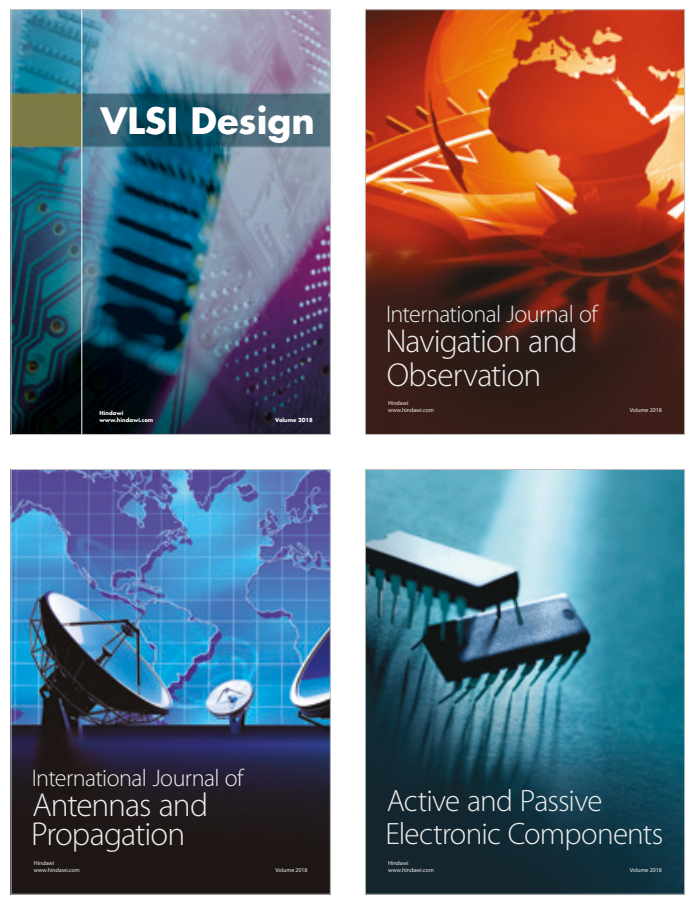
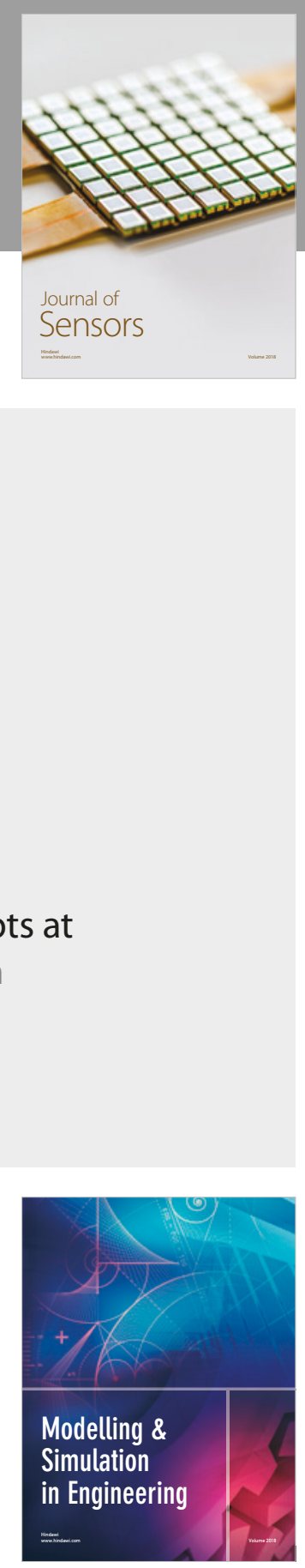

\section{Advances \\ Multimedia}
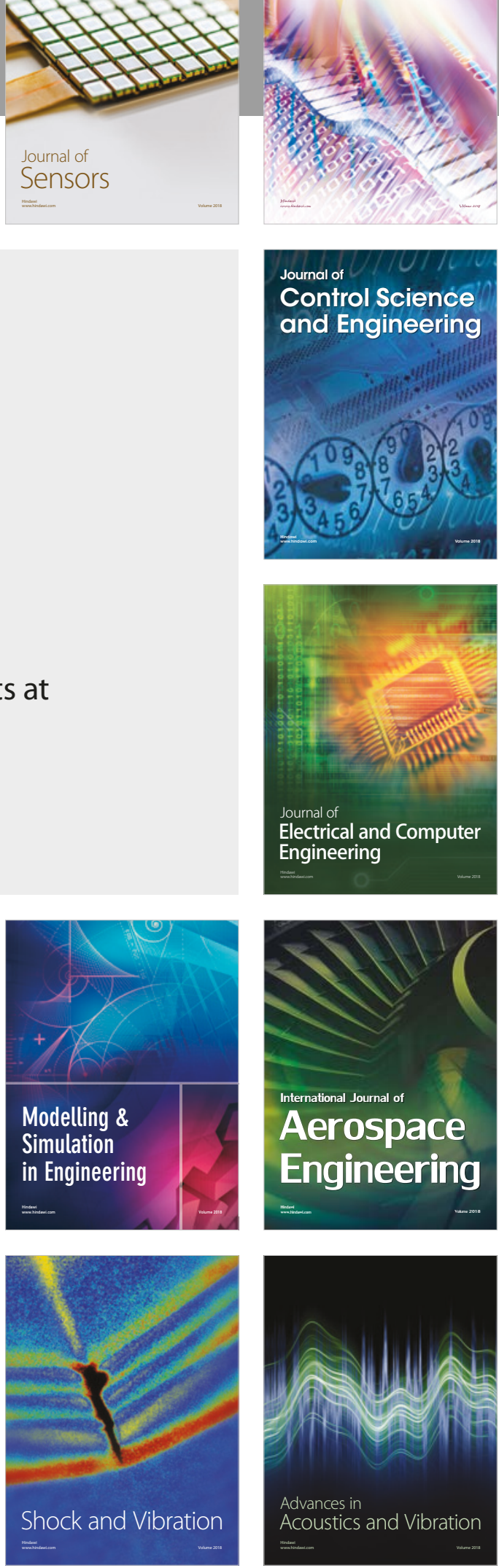